\title{
The Use of the Nursing Interventions Classification in Identifying the Workload of a Nursing Team in a Pediatric Oncology Center
}

\author{
Jiwen SUN ${ }^{\mathrm{a}}$, Yihan $\mathrm{LI}^{\mathrm{a}}$ and Nanping Shen ${ }^{\mathrm{a}, 1}$ \\ a'Shanghai Children's Medical Center
}

\begin{abstract}
The activities/interventions performed by the nursing team and the time required for their performance have become a widely discussed topic due to their impact on the quality of patient safety and staff working conditions. Nursing Interventions Classification (NIC) was used as a more accurate indicator as it identified activities/interventions. This study analyzed the workload distribution of nursing team in a pediatric oncology center.
\end{abstract}

Keywords. Nursing, nursing interventions classification, work sampling, workload

\section{Introduction}

Nursing Interventions Classification (NIC) have been used as a basis for measuring workload and an important resource in supporting workforce planning[1]. Nursing workforce size have been a source of conflict among managers[2]. The aim of our study is to identify and analyze the distribution of nursing professionals' workloads, according to the NIC in a pediatric oncology center.

\section{Approach}

This was an observational study. The sample consisted of 17 nurses who performed a variety of roles within pediatric oncology center. A list of pediatric oncology nursing interventions and a work sampling instrument were formed according to NIC taxonomy and used to conduct a work sampling study, in which, the researcher observed all the nurses or assistants on duty for 30 days, and 8 hours for each day (8:00-17:00), and recorded a snapshot of nursing activities at 10 minute intervals according to work sampling theory.

${ }^{1}$ Corresponding Author, Nanping Shen, Shanghai Children's Medical Center, 1678 Dongfang Road, Pudong District, Shanghai, China; E-mail: shennanping@scmc.com.cn. 


\section{Results}

A total of 13021 activities were identified and mapped into 89 nursing interventions, 63 were classified as direct care interventions and 26 as indirect care interventions, in accordance with the NIC.

The most representative domains were Health System (58.76\%) and PhysiologicalComplex (29.16\%). The pediatric oncology nurses' working time was categorized into $35.84 \%$ of direct-care interventions, $43.66 \%$ of indirect - care interventions, $10.82 \%$ of unit-related activities and $9.68 \%$ of non-productive activities. The productivity of the nursing team reached $90.32 \%$.

\section{Conclusions}

Identification and classification of nursing interventions and activities, according to the NIC, and measurement of frequencies through the work sampling technique of this study, enabled the assessment of workload distribution of the pediatric oncology nurses.

\section{References}

[1] da Cruz CW, Bonfim D, Gaidzinski RR, Fugulin FM, Laus AM. The use of Nursing Interventions Classification (NIC) in identifying the workload of nursing: an integrative review. Int J Nurs Knowl 25 (2014), 154-160.

[2] de Cordova PB, Lucero RJ, Hyun S, Quinlan P, Price K, Stone PW. Using the nursing interventions classification as a potential measure of nurse workload. J Nurs Care Qual 25 (2010), 39-45. 